\title{
DISTRIBUCIÓN ECOLÓGICA DE LA HERPETOFAUNA EN GRADIENTES ALTITUDINALES SUPERIORES DEL CERRO EL POTOSI, GALEANA, NUEVO LEÓN, MÉXICO
}

\author{
Jorge A. Contreras-Lozano, ${ }^{1}$ David Lazcano ${ }^{1}$ y Armando J. \\ CONTRERAS-BALderas ${ }^{2}$ \\ ${ }^{1}$ Laboratorio de Herpetología y ${ }^{2}$ Laboratorio de Ornitología, Facultad de Ciencias Biológicas, \\ Universidad Autónoma de Nuevo León. Apartado Postal 513 y 425. San Nicolás de los Garza, \\ Nuevo León. México C.P.66450.<pichi_best@hotmail.com; dlazcanov@hotmail.com; \\ arcontre@fcb.uanl.mx>
}

Contreras-Lozano, J. A., D. Lazcano \& A. J. Contreras-Balderas. 2011. Distribución ecológica de la herpetofauna en gradientes altitudinales superiores del Cerro El Potosí, Galeana, Nuevo León, México. Acta Zoológica Mexicana (n. s.), 27(2): 231-243.

RESUMEN. Este estudio herpetofaunístico se realizó en el Cerro El Potosí en el municipio de Galeana, Nuevo León, México, entre noviembre de 2006 y noviembre de 2007. Este es el primer trabajo formal extensivo que se realiza en esta área, lo que permite incrementar el conocimiento de la herpetofauna de la Sierra Madre Oriental y en particular de esta montaña, un Área Natural Protegida a nivel estatal, y aportar información que permita establecer mejores estrategias de manejo y conservación de este grupo de vertebrados. El Cerro El Potosí cuenta con un gradiente altitudinal que va desde los 2000 hasta los $3750 \mathrm{msnm}$, donde se presentan distintas comunidades vegetales. Los objetivos del trabajo fueron: determinar las especies que ocurren en el gradiente altitudinal, determinar sus preferencias de sustrato, y su distribución en diferentes comunidades vegetales. Los muestreos se realizaron mediante transectos, siguiendo el método de inventario y muestreo de Campbell y Christman (1982). Se capturaron 188 individuos de siete especies, una especie de anuro, una especie de urodelo, cuatro especies de saurios y una especie de serpiente.

Palabras Clave: Cerro el Potosí, Distribución, Herpetofauna, Galeana, Nuevo León, México.

Contreras-Lozano, J. A., D. Lazcano \& A. J. Contreras-Balderas. 2011. Ecological distribution of the herpetofauna in top gradients in Cerro El Potosi, Galeana, Nuevo Leon, Mexico. Acta Zoológica Mexicana (n. s.), 27(2): 231-243.

ABSTRACT. This herpetofaunistic study was conducted in Cerro El Potosi in the municipality of Galeana, Mexico, between November 2006 and November 2007. This is the first extensive formal work done in the area, thus increasing the knowledge of the herpetofauna of the Sierra Madre Oriental and in particular of this mountain, a State Protected Natural Area, and providing information that will enhance management and conservation strategies for this vertebrate group. Cerro El Potosí has an altitude gradient between 2000 and $3750 \mathrm{~m}$, where distinct plant communities are found. The objectives of this re-

Recibido: 30/07/2009; aceptado: 14/12/2010. 
search were to identify species that occur in the altitudinal gradient, as well as their substrate preferences and distribution in different plant communities. Sampling was performed using transects, following the sampling and inventory method of Campbell and Christman (1982). A total of 188 individuals of seven species were collected, one species of frog, one species of salamander, four species of lizards and one species of snake.

Key word: Cerro El Potosí, Distribution, Herpetofauna, Galeana, Nuevo Leon, Mexico.

\section{INTRODUCCIÓN}

Al igual que la flora, la fauna de México es también una de las más ricas del mundo. La fauna de anfibios y reptiles cuenta con aproximadamente 1627 especies en toda la extensión el país (Liner 2007), sobresaliendo el elevado endemismo que presenta en comparación con la herpetofauna de otros países. Las especies reconocidas como amenazadas o en peligro de extinción en México hasta 1994 sumaban un total de 234 lo que representaba un $7.7 \%$ del total registrado para el país en ese entonces (FloresVillela \& Gerez 1994).

La herpetofauna conocida para la Sierra Madre Oriental comprende 207 especies. Esta incluye 20 especies de salamandras, 44 especies de anuros, 49 saurios, 88 serpientes y 6 especies de tortugas (Canseco-Márquez et al. 2004).

Varios trabajos han sido importantes para el estudio de la herpetofauna en el estado de Nuevo León. Martín del Campo (1949), realizó una revisión de datos herpetológicos para el estado; Aseff (1967) registró 45 especies, nueve de anfibios y 36 de reptiles para el centro, sur y norte del estado, de las cuales cinco especies fueron registradas por primera vez; para el municipio de Galeana, Horowitz (1955) reconoció la presencia de Phrynosoma orbiculare, Knight y Scudday (1985) registraron la presencia de Gerrhonotus parvus y Treviño (1978) realizó un inventario herpetológico con el que determinó 57 especies y subespecies, y siete fueron nuevos registros; ninguno de los autores anteriores documentó o realizó colectas continuas para el Cerro El Potosí. De la Sierra San Antonio Peña Nevada, Taylor (1941) describió una nueva especie de pletodóntido de Pseudoeurycea galeanae, que ocupa hábitats terrestres y semiárboreos, y Rabb (1956) describió una nueva especie de salamandra, Chiropterotriton priscus.

Los estudios de la herpetofauna en áreas montañosas en el estado de Nuevo León se han incrementado desde que se establecieron áreas naturales protegidas nacionales (Anónimo 2000, Arriaga et al. 2000), como el Cerro El Potosí (Anónimo 2000), el Parque Ecológico Chipinque (Banda 2002, Lazcano et al. 2006), la Sierra San Antonio Peña Nevada (Lazcano et al. 2004), la Sierra Cerro de la Silla (Gallardo-Valdez 2006, Lazcano et al. 2009) y la Sierra de Picachos (Contreras-Lozano et al. 2007), todas en la Sierra Madre Oriental.

El presente estudio herpetofaunístico se realizó en el Área Natural Protegida Cerro El Potosí, Galeana, Nuevo León, en la Sierra Madre Oriental. Este es el primero 
que se realiza sobre la herpetofauna del área con base en muestreos en campo, para incrementar el conocimiento de la herpetofauna del estado y aportar información para establecer mejores estrategias de manejo y conservación de este grupo de vertebrados. Los objetivos de este estudio fueron determinar las especies de anfibios y reptiles que ocurren en un gradiente altitudinal, sus preferencias de sustrato y su distribución en diferentes comunidades vegetales.

\section{MATERIALES Y MÉTODOS}

Área de estudio. El Cerro El Potosí es parte de la Sierra Madre Oriental y se ubica en el centro sur del Estado de Nuevo León a $15 \mathrm{~km}$ al oeste de la cabecera municipal de Galena, entre los $24^{\circ} 50^{\prime} 35^{\prime \prime}$ y $24^{\circ} 53^{\prime} 16^{\prime \prime} \mathrm{N}$ y $\operatorname{los} 100^{\circ} 13^{\prime} 9^{\prime \prime}$ y $100^{\circ} 15^{\prime} 12^{\prime \prime}$ W (García-Arévalo y González-Elizondo 1991) (Figura 1). El gradiente altitudinal de este cerro va de los 2000 a los 3750 msnm.

De acuerdo con la clasificación climática de Köppen modificada por García (1981), el clima del área de estudio corresponde al tipo $\mathrm{E}(\mathrm{T}) \mathrm{H}(\mathrm{e})$ con temperatura media del mes mas caliente menor a $10^{\circ} \mathrm{C}$; por esta temperatura se le ubica como cli-

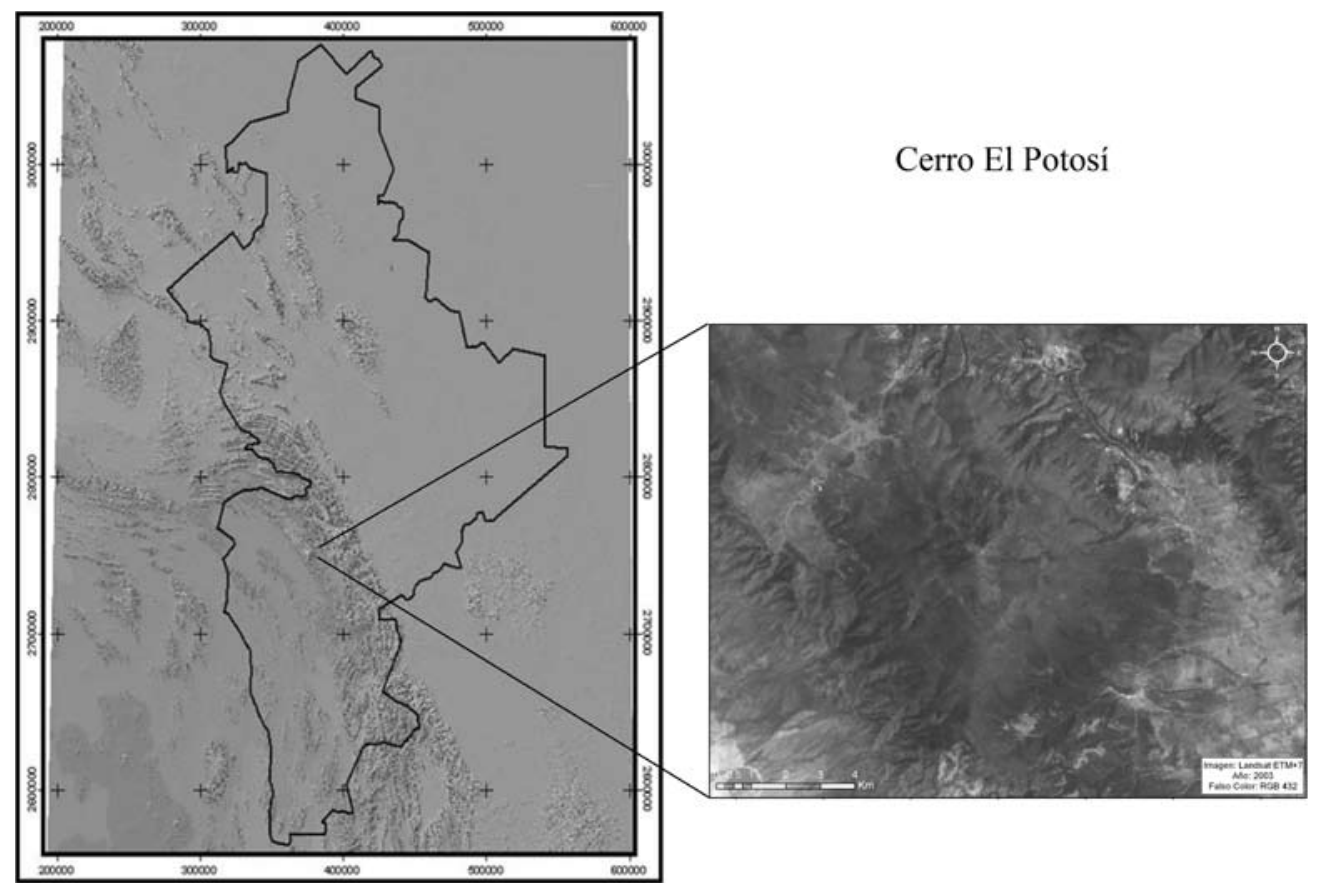

Figura 1. Localización del Cerro El Potosí en el municipio de Galeana, Nuevo León. 
ma de tundra, aunque el término no es válido al estar esta área en latitudes inferiores. Este clima se presenta en altitudes por encima de los $1500 \mathrm{msnm}$ y se le considera extremoso por la oscilación de temperatura entre $7^{\circ}$ y $14^{\circ} \mathrm{C}$ entre las medias del mes más frío y del mes más caliente del año. En el área de estudio esa oscilación es de $8.7^{\circ}$ $\mathrm{C}$ y con una precipitación media anual de $27.4 \mathrm{~mm}$. La Figura 2 muestra la precipitación mensual durante la época de muestreo (noviembre 2006-2007) obtenido de la Comisión Nacional del Agua (CONAGUA 2010).

Por su altitud el Cerro El Potosí es considerado como un parteaguas de las tres regiones hidrológicas de Nuevo León (Río Bravo, San Fernando-Soto la Marina y El Salado). Tomando como referencia la cima las escorrentías principales son: arroyo el Agua (al norte), arroyo las Ardillas (al noreste), arroyo el Alquitrán (al noroeste), arroyo Labores de la Verdia (al suroeste), arroyo Santa Teresa (al sur), arroyo Chupaderos (al sur) y arroyo el Barro (al sur) (INEGI 1986).

Siguiendo los criterios de García-Arana (1996) en el área se encuentran los siguientes tipos de vegetación en diferentes intervalos altitudinales: matorral mediano esclerófilo de Quercus intricata entre 2000-2200 msnm, que en la misma área se asocia con áreas de cultivo, bosque de Pinus cembroides entre 2200 y $2500 \mathrm{msnm}$, bosque de Pinus ayacahuite entre 2500 y $3500 \mathrm{msnm}$, bosque de Pinus hartwegii entre 2900 y $3000 \mathrm{msnm}$, matorral de Pinus culminicola entre 3100 y $3650 \mathrm{msnm}$ y pradera subalpina entre 3600 y $3715 \mathrm{msnm}$.

Muestreo de la herpetofauna. Se realizaron transectos en las comunidades vegetales de Pinus ayacahuite, P. pseudostrobus, Pseudotsuga menziesii, Pinus hartwegii,

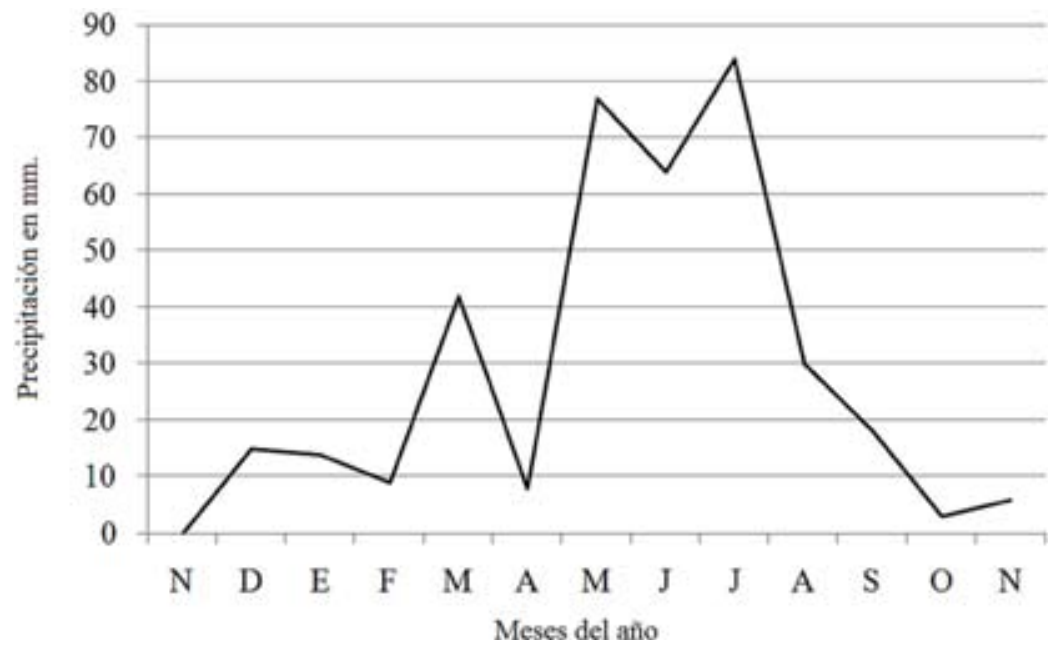

Figura 2. Gráfico de la precipitación durante el periodo de estudio noviembre 2006- noviembre 2007 en el Cerro El Potosí, Galeana, Nuevo León. 
P. strobiformis y P. culminicola, abarcando los gradientes altitudinales de 2800 a $3750 \mathrm{msnm}$. Una vez determinadas estas comunidades se realizaron los transectos mediante el método de inventario y muestreo descrito por Campbell \& Christman (1982), que consiste en localizar y capturar los ejemplares en el sustrato que estaba siendo utilizado ya sea debajo o sobre las piedras, troncos, vegetación y en sustratos artificiales (paredes, láminas, etc.), los transectos fueron de $1000 \mathrm{~m}$ por $6 \mathrm{~m}$ de ancho a cada lado del transecto. Se realizaron 12 muestreos de campo entre noviembre de 2006 y noviembre de 2007, el horario de captura fue de 8:00 a 14:00 y 15:00 a 19:00h durante tres días, dando $30 \mathrm{~h}$ esfuerzo/hombre en cada sesión de muestreo. Todos los ejemplares fueron depositados en la Colección del Laboratorio de Herpetología de la FCB_UANL. Los ejemplares recolectados fueron identificados, utilizando los criterios de Smith \& Taylor (1945,1950 y 1966) y de Conant \& Collins (1998). La preferencia de microhábitat de cada especie se determinó haciendo la observación del tipo de sustrato en el que se encontró y el tipo de vegetación, tomando como base el estrato arbóreo más predominante en el área en que se encontraron los individuos. Los nombres científicos y comunes están basados en Crother et al. (2003), FloresVillela \& Canseco-Márquez (2004) y Frost et al. (2006).

Análisis de datos. Se graficó la distribución altitudinal de las especies registradas, mostrando los intervalos en los que se encuentran y el número de especies para cada intervalo.

Se elaboró una curva de acumulación de especies utilizando el programa EstimateS 7 (Colwell 2004), por medio de los estimadores no paramétricos de diversidad Chaol y Jacknife1 que indican cuantas especies pueden ser incorporadas de acuerdo al número de muestreos.

\section{RESULTADOS}

Se recolectaron siete especies en el Cerro El Potosí, la distribución de acuerdo a la comunidad vegetal y altitud se observa en los Cuadros 1 y 2 . La curva de acumulación de especies arrojó valores de Chao $1=8$ y Jacknife $1=8.83$, alcanzando la asíntota en el gráfico (Figura 3).

En el intervalo altitudinal de 3100-3300 msnm se presentaron siete especies y la cima (3600-3750 msnm) presentó menor riqueza específica, con sólo tres especies (Figura 4).

Cuatro especies se encuentran enlistadas en la NOM-059-SEMARNAT-2008 de las cuales Pseudoeurycea galeanae, Barisia ciliaris, Sceloporus grammicus y Crotalus pricei están consideradas bajo protección especial.

El microhábitat y tipos de vegetación en los que se encontraron las especies se describen a continuación. La distribución altitudinal de cada especie se describe en el Cuadro 2. 


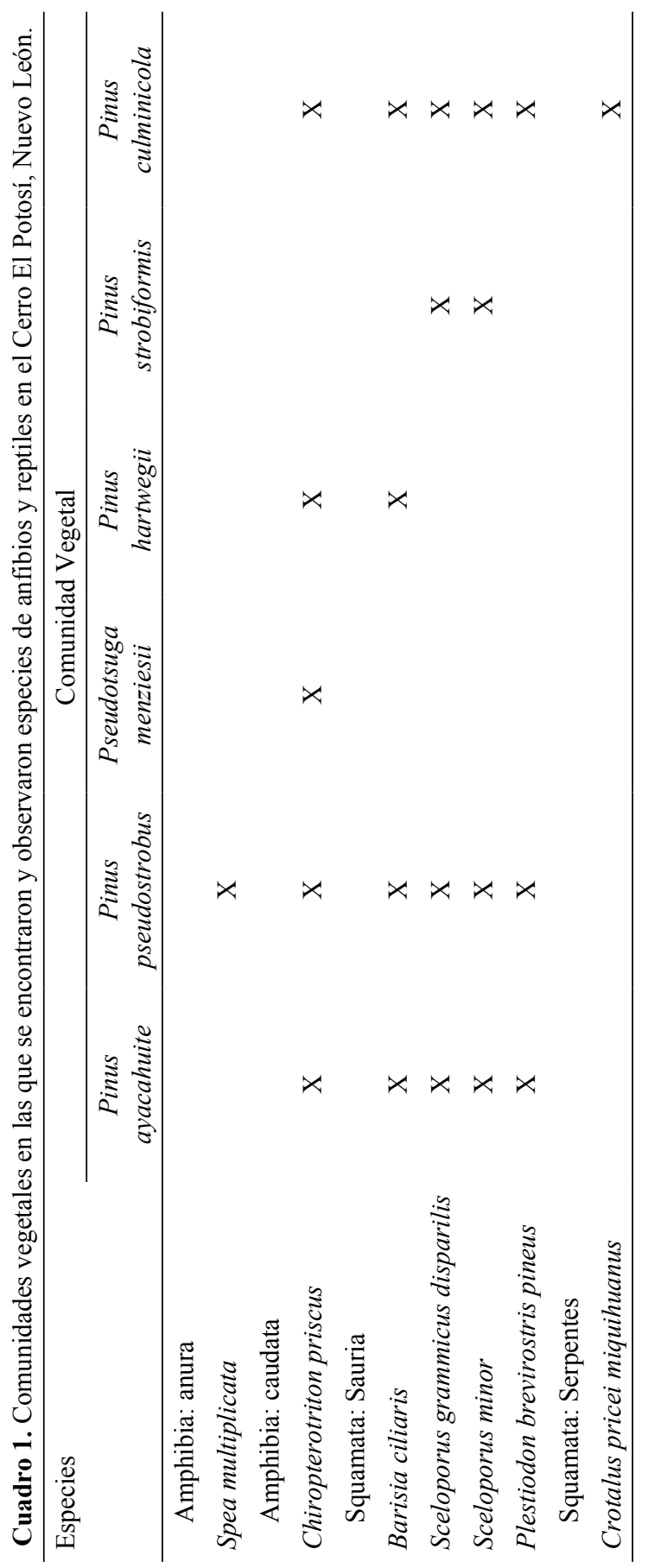


Cuadro 2. Especies encontradas en el Cerro El Potosí, intervalo altitudinal y el estatus correspondiente en la Norma Oficial Mexicana (NOM-059-ECOL-2008). Pr = Protección Especial; SE = Sin Estatus.

\begin{tabular}{lcc}
\hline Especies & $\begin{array}{c}\text { Altitud Min-max } \\
(\mathrm{msnm})\end{array}$ & Estatus \\
\hline Amphibia: anura & 3293 & $\mathrm{SE}$ \\
$\begin{array}{l}\text { Spea multiplicata } \\
\quad \text { Amphibia: caudata }\end{array}$ & & $\mathrm{Pr}$ \\
Chiropterotriton priscus & $2281-3479$ & \\
$\quad$ Squamata: Sauria & & $\mathrm{Pr}$ \\
Barisia ciliaris & $3218-3293$ & $\mathrm{Pr}$ \\
Sceloporus grammicus disparilis & $3233-3725$ & $\mathrm{SE}$ \\
Sceloporus minor & $2909-3641$ & $\mathrm{SE}$ \\
Plestiodon brevirostris pineus & $3114-3339$ & \\
$\quad$ Squamata: Serpentes & & $\mathrm{Pr}$ \\
Crotalus pricei miquihuanus & $2933-3305$ & \\
\hline
\end{tabular}

Spea multiplicata. Observado una sola vez debajo de una piedra en bosque de Pinus pseudostrobus.

Chiropterotriton priscus. Ocurre en las partes altas del cerro (37 individuos observados) y restringida a las partes húmedas que presentan bosque de pino. Prefiere sustratos como piedras, troncos secos y raíces.

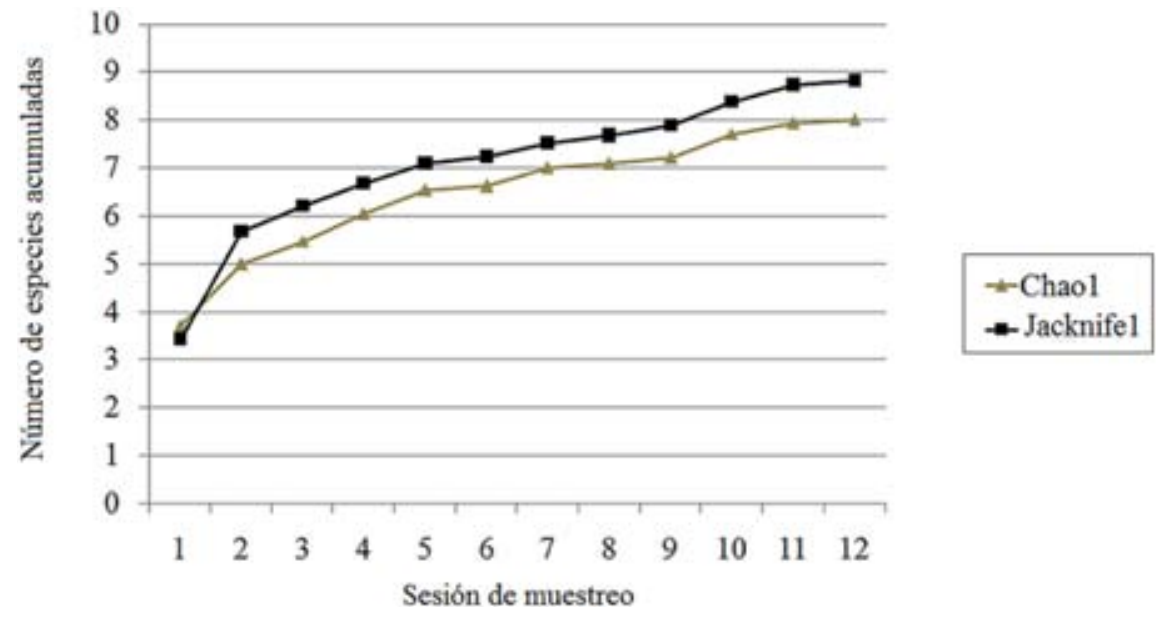

Figura 3. Curva de acumulación de especies de anfibios y reptiles durante noviembre 2006noviembre 2007 en el Cerro El Potosí, Nuevo León, entre los 2800 y 3750 m de altitud. 


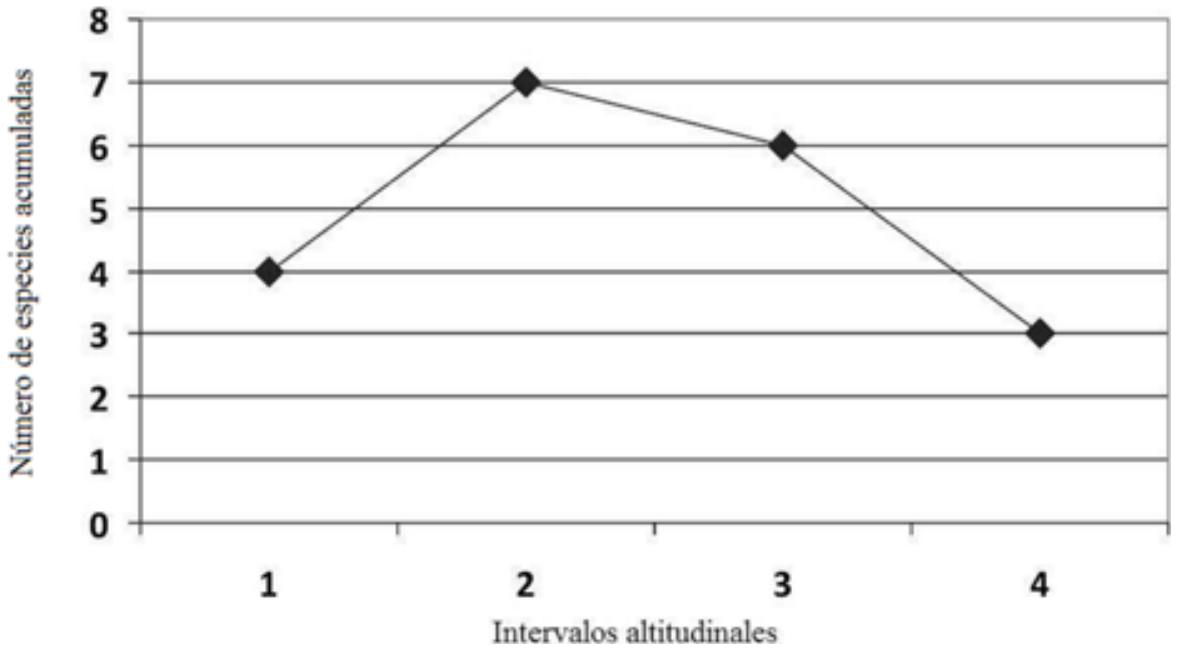

Figura 4. Número de especies de anfibios y reptiles en cuatro intervalos altitudinales (msnm) del Cerro El Potosí, Nuevo León (1=2800-3100 m, 2=3100-3300m, 3=3300-3600 m, 4=3600-3750 m).

Barisia ciliaris. Se observaron 14 individuos. Muestra preferencia por estar debajo de las piedras y troncos secos.

Sceloporus grammicus disparilis. Se observaron 41 individuos. Esta especie ocupó diferentes sustratos como paredes de piedra, troncos y agaves, se distribuye en las comunidades de bosque de pino, bosque de encino, e incluso es de los más abundantes en zonas que han sido previamente incendiadas.

Sceloporus minor. Se observaron 71 individuos, principalmente en lugares pedregosos y con alta incidencia de la luz solar. Presente en el bosque de pino y bosque de encino.

Plestiodon brevirostris pineus. Se observaron 19 individuos en el bosque de pino, donde muestran preferencia por estar debajo de piedras y troncos secos.

Crotalus pricei miquihuanus. Se observaron seis individuos en el bosque de pino. Está presente en ambientes de áreas pedregosas y agaves.

\section{DISCUSIÓN}

Este trabajo aporta información sobre la presencia de siete especies para la cima del Cerro El Potosí, de los cuales Spea multiplicata es un nuevo registro.

La curva de acumulación de especies alcanzó una asíntota por lo que el número de muestreos fue adecuado (Colwell \& Coddington 1994). Según los valores de los dos estimadores paramétricos $($ Chao $1=8$, Jack $1=8.83)$ se registró el $80 \%$ de la herpetofauna para el área estudiada. La riqueza especifica de reptiles y anfibios fue mayor en 
la comunidad de Pinus pseudostrobus y P. culminicola en donde se observaron seis de las siete especies, mientras que la menor riqueza especifica ocurrió en la comunidad de Pseudotsuga menziesii, con una especie observada. En el documento oficial de las Áreas Naturales Protegidas de Nuevo León (Anónimo 2000) se menciona con base en consultas bibliográficas que en el Cerro El Potosí existen 33 especies de reptiles y ocho especies de anfibios, sin especificar el gradiente altitudinal que ocupa cada especie. Dicho documento no incluye a Sceloporus grammicus disparilis, Sceloporus minor, Crotalus pricei miquihuanus y Spea multiplicata, cuya presencia fue confirmada en este estudio. Behler \& King (1992), Conant \& Collins (1998), Werler \& Dixon (2000), Köhler \& Heimes (2002), Canseco-Márquez et al. (2004), Dixon \& Werler (2005) y Lemos \& Smith (2007) mencionaron como probable la presencia de las siete especies observadas en el área de estudio, con este trabajo se ratificó su presencia.

En estudios herpetológicos recientes en áreas montañosas de Nuevo León se ha reportado la siguiente riqueza específica: para el Parque Ecológico Chipinque en el municipio Garza García (Lazcano et al. 2006) 45 especies; en la Sierra Cerro de la Silla en el municipio Cadereyta-Jiménez (Gallardo-Valdez 2006, Lazcano et al. 2009) 43 especies; para la Sierra San Antonio Peña Nevada en el municipio de Zaragoza (Lazcano et al. 2004) 32 especies; y en la Sierra de Picachos en la Sierra Madre Oriental (Contreras-Lozano et al. 2007) 33 especies. La baja diversidad encontrada en el Cerro el Potosí (siete especies) se debe al intervalo altitudinal explorado.

En el intervalo altitudinal de 2800-3100 msnm se observaron cuatro especies, en el intervalo 3100-3300 msnm se observaron siete especies y el número de especies disminuyó a tres en la cima. Se ha demostrado que la diversidad faunística tiende a disminuir conforme la altitud es mayor (Daniels 1992, Patterson et al. 1998).

Cuatro especies (57.14\%), Pseudoeurycea galeanae, Barisia ciliaris, Sceloporus grammicus y Crotalus pricei de las siete presentes en el Cerro el Potosí están enlistadas en la NOM-059-SEMARNAT-2008 en la categoría bajo protección especial, por lo que es importante preservar los hábitats en los gradientes altitudinales muestreados para la supervivencia de estos taxa.

La presencia de Chiropterotriton priscus en el Cerro El Potosí previamente documentada (Treviño 1978) se corrobora con este estudio, no obstante que el hábitat del que depende esta salamandra ha sido muy alterado por los efectos de incendios forestales (en los años 1972 y 1998), deforestación, introducción de ganado y campos de cultivo. Estos efectos se han observado a partir de los trabajos de García-Arévalo \& González-Elizondo (1991) y Beaman \& Andresen (1966) y hasta la fecha no se ha documentado sobre la pérdida de hábitat e introducción de ganado que se ha realizado en este cerro.

Para el Cerro El Potosí, sin mencionar el gradiente altitudinal en el que fueron colectadas, se encuentran preservadas en la colección herpetológica del Laboratorio 
de Herpetología, Facultad de Ciencias Biológicas, UANL, las especies : Chiropterotriton priscus, Lithobates berlandieri, Barisia imbricata ciliaris, Gerrhonotus infernalis, Sceloporus grammicus disparilis, Sceloporus jarrovi minor, Scincella silvicola caudaequinae y Natrix valida valida ahora llamada Thamnophis valudis validus (Liner 2007) incrementándose a 11 las especies presentes para el Cerro el Potosí.

Spea multiplicata fue la especie menos observada en este estudio, probablemente por el gradiente altitudinal en el que se encontró (3293 msnm); Canseco-Márquez et al. (2004) documentó esta especie en la Sierra Madre Oriental entre 1600 y 2000 msnm. En el área de estudio el único individuo de esta especie se observó en el mes de julio, durante la época lluviosa (Fig. 2).

Los efectos negativos de la fragmentación de hábitat sobre las especies han sido ampliamente reconocidos (Gilpin \& Soulé 1986, Lovejoy et al. 1986, Wilcove et al. 1986). Una acción común para la disminución de pérdida de hábitat y la fragmentación es establecer parques o reservas, pero incluso en estas áreas la recreación humana puede impactar negativamente la biota protegida (Boyle \& Samson 1985, Pomerantz et al. 1988, Garber \& Burger 1995).

Es necesario realizar más trabajos de conservación en el área, ya que está siendo fuertemente impactado por la tala inmoderada y los incendios registrados en décadas anteriores, y aparentemente no presenta una recuperación del hábitat (Anónimo 2000).

AGRADECIMIENTOS. Queremos agradecer a la Universidad Autónoma de Nuevo León y CONACyT. El financiamiento al proyecto PAICYT CN-1371-06 titulado "Estado actual de la fauna silvestre en el Cerro El Potosí, Área Natural Protegida de Nuevo León, México”. A la SEMARNAT por el permiso de colecta Oficio Num. SGPA/DGVS/01732/08. También a las personas participantes en este proyecto Dr. José Ma. Torres Ayala, y los compañeros: Jesús Leza, Fernando Solís, Cuauhtémoc Ibarra y Liz Gaspar. A los árbitros por su valioso apoyo para mejorar el manuscrito.

\section{LITERATURA CITADA}

Anónimo. 2000. Decretos de áreas naturales del estado de Nuevo León, México. Secretaría de Ecología y Recursos Naturales. Periódico Oficial 2000.

Arriaga, L., J. M. Espinosa, C. Aguilar, E. Martínez, L. Gómez \& E. Loa (coordinadores) 2000. Regiones Terrestres Prioritarias de México. Comisión Nacional para el Conocimiento y Uso de la Biodiversidad. México, D.F.

Aseff, A. 1967. Notas sobre la Herpetofauna del Centro de Nuevo León, México. Tesis de Licenciatura. Universidad Autónoma de Nuevo León. Facultad de Ciencia Biológicas. Monterrey, Nuevo León.

Banda, J. L. 2002. Aspectos Ecológicos de la Herpetofauna del Parque Ecológico Chipinque, Ubicado en los Municipios de Garza García y Monterrey, Nuevo León, México. Universidad Autónoma de Nuevo León. Facultad de Ciencias Biológicas. Monterrey, Nuevo León.

Beaman, J. H. \& J. W. Andresen. 1966. The vegetation, floristics and phytogeography of the summit of Cerro Potosi, Mexico. The American Midland Naturalist, 75: 1-33.

Behler, J. L. \& W. King. 1992. The Audubon Society field guide to North American reptiles and amphibians. Chanticleer Press, Inc. N.Y. 
Boyle, S. \& F. B. Samson. 1985. Effects of nonconsumptive recreation on wildlife: a review. Wildlife Society Bulletin, 13: 110-116.

Campbell, H. W. \& S. P. Christman. 1982. Field techniques for herpetofaunal community analysis. Pp. 193-200. In: N.J. Scott, Jr. (Ed). Herpetological Communities. U. S. Fish and Wildlife Service. Wildlife Research Report 13.

Canseco-Márquez, L., F. Mendoza-Quijano \& G. Gutiérrez-Mayén. 2004. Análisis de la Distribución de la Herpetofauna, pp. 417-437. In: I. Luna, J.J. Morrone y D. Espinoza (Eds). Biodiversidad de la Sierra Madre Oriental. Las Prensas de Ciencias. México, D.F.

Colwell, R. K. 2004. EstimateS: Statistical Estimation of Species Richness and Shared Species for Samples. Version 7. Aplicación en línea en http://viceroy.eeb.uconn.edu/estimates

Colwell, R. K. \& J. A. Coddington. 1994. Estimating terrestrial biodiversity through extrapolation. Philosophical Transactions of the Royal Society, 345: 101-118.

CONAGUA. 2010. Base de datos de estaciones climatológicas del Servicio Meteorológico Nacional, México.

Conant, R. \& J. T. Collins. 1998. A field guide to reptiles and amphibians of Eastern and Central North America. $3^{\text {rd }}$. Edition. Houghton Mifflin Co. Boston.

Contreras-Arquieta, A. \& D. Lazcano. 1995. Lista revisada de los reptiles del estado de Nuevo León, México. Pp. 57-64. In: Contreras-Balderas, S., F. González, D. Lazcano \& A.J. Contreras-Balderas (Eds.). Listado Preliminar de la Fauna Silvestre del Estado de Nuevo León, México. Consejo Consultivo para la Preservación y Fomento de la Flora y Fauna Silvestre de Nuevo León. Monterrey, Nuevo León.

Contreras-Lozano, J. A., D. Lazcano \& A. J. Contreras-Balderas. 2007. Notes on Mexican Herpetofauna 10: The Herpetofauna of Three Plant Communities in the Sierra de Picachos, Nuevo León, México. Bulletin Chicago Herpetological Society, 42: 177-182.

Crother, B. I., J. Boundy, J. A. Campbell, K. De Quieroz, D. Frost, D. M. Green, R. Highton, J. B. Iverson, R. W. McDiarmid, P. A. Meylan, T. W. Reeder, M. E. Seidel., J. W. Sites, Jr., S. G. Tilley \& D. B. Wake. 2003. Scientific and Standard English Names of Amphibians and Reptiles of North America North of Mexico: Update. Herpetological Review, 34: 193-206.

Daniels, R. J. R. 1992. Geographic distribution patterns of amphibians in the western Ghats, India. Journal of Biogeography, 19: 521-529.

Dixon, J. R. \& J. E. Werler. 2005. Texas snakes: A field guide. University of Texas Press. Austin, Texas.

Flores-Villela, O. \& P. Gerez. 1994. Biodiversidad y conservación en México: Vertebrados, vegetación y uso del suelo. CONABIO, UNAM. México, D.F.

Flores-Villela, O. \& L. Canseco-Márquez. 2004. Nuevas Especies y Cambios Taxonómicos para la Herpetofauna de México. Acta Zoológica Mexicana (n.s.), 20: 115-144.

Frost, D. R., T. Grant, J. N. Faivovich, R. H. Bain, A. Haas, C. F. B. Haddad, R. O. de SA, A. Channing, M. Willkinson, S. C. Donnellan, C. J. Raxworthy, J. A. Campbell, B. L. Blotto, P. Moler, R. C. Drews, R. A. Nussbaum, J. D. Lynch, D. M. Green \& W. C. Wheeler. 2006. The Amphibian Tree of Life. Bulletin of the American Museum of Natural History, 297: 1-370.

Gallardo-Valdez, J. 2006. Distribución de la herpetofauna en las diferentes comunidades de vegetación de las localidades "Boquillas y Atongo" del municipio de Cadereyta, dentro del área natural protegida Sierra de Cerro la Silla, Nuevo León, México. Tesis de Licenciatura. Universidad Autónoma de Nuevo León. Facultad de Ciencias Biológicas. Monterrey, Nuevo León.

Garber, S. D. \& J. Burger. 1995. A 20-year study documenting the relationship between turtle decline and human recreation. Ecological Applications, 5: 1151-1162. 
García-Arana, M. A. 1996. Análisis de la cubierta vegetal y propuesta para la zonificación ecológica del Cerro El Potosí, Galeana, Nuevo León, México. Universidad Autónoma de Nuevo León. Facultad de Ciencias Forestales. Linares, Nuevo León.

García-Arévalo, A. \& S. González-Elizondo. 1991. Flora y vegetación de la cima del Cerro Potosí, Nuevo León, México. Acta Botánica Mexicana, 13: 53-74.

García, E. 1981. Modificación al Sistema de Clasificación Climática de Köppen. Instituto de Geología, UNAM, $3^{\text {a }}$ Edición. México, D.F.

Gilpin, M. E. \& M. E. Soulé. 1986. Minimum viable populations: processes of species extinction. Pp. 19-34. In: M.E. Soulé (Ed.). Conservation Biology: the Science of Scarcity and Diversity. Sinauer Associates. Sunderlands, MA.

Horowitz, S.B. 1955. An Arrangement of the Subspecies of the Horned Toad, Phrynosoma orbiculare (Iguanidae). The American Midland Naturalist, 54: 204-218.

INEGI. 1986. Síntesis Geográfica del Estado de Nuevo León, Instituto Nacional de Estadística Geográfica e Informática. Instituto de Geografía, UNAM. Cartas de Climas. Monterrey 14R-VII S. P.

Knight, R. A. \& J. F. Scudday. 1985. A New Gerrhonotus (Lacertilia:Anguidae) from the Sierra Madre Oriental, Nuevo León, México. The Southwestern Naturalist, 30: 89-94.

Köhler, G. \& P. Heimes. 2002. Stachelleguane, Lebensweise, Pflege, Zucht. Offenbach: Herpeton.

Lazcano, D., J. Banda, G. Castañeda, C. García-de la Peña \& C. Solís-Rojas. 2006. Notes on Herpetofauna 8: Herpetofauna of the Parque Ecológico Chipinque, Nuevo León, México. Bulletin Chicago Herpetological Society, 41: 117-123.

Lazcano, D. \& A. Contreras-Arquieta. 1995. Lista revisada de los anfibios del estado de Nuevo León, México. Pp. 67-70. In: Contreras-Balderas, S., F. González, D. Lazcano \& A.J. Contreras-Balderas (Eds.). Listado Preliminar de la Fauna Silvestre del Estado de Nuevo León, México. Consejo Consultivo para la Preservación y Fomento de la Flora y Fauna Silvestre de Nuevo León. Monterrey, Nuevo León.

Lazcano, D., A. Contreras- Balderas, J. I. González- Rojas, G. Castañeda, C. García-de la Peña \& C. Solís- Rojas. 2004. Notes on Herpetofauna 6: Herpetofauna of Sierra San Antonio Peña Nevada, Zaragoza, Nuevo León, México: Preliminary List. Bulletin of the Chicago Herpetological Society, 39: 181-187.

Lazcano, D., J. A. Contreras-Lozano, J. Gallardo-Valdez, C. García- de la Peña, \& G. Castañeda. 2009. Notes on Mexican Herpetofauna 11: Herpetological Diversity in Sierra Cerro de La Silla (Saddleback Mountain), Nuevo León, México. Bulletin Chicago Herpetological Society, 44: 2127.

Lemos-Espinal, J. A. \& H. M. Smith. 2007. Anfibios y Reptiles del Estado de Coahuila, México. UNAM, CONABIO. México, D.F.

Liner, E. A. 2007. A Checklist of the Amphibians and Reptiles of Mexico. Occasional Papers of the Museum of Natural Science, Louisiana State University, 80: 1-59.

Lovejoy, T. E., R. O. Bierregaard Jr., A. B. Rylands, J. R. Malcom, C. E. Quintela, L. H. Harper, K. S. Brown Jr., A. H. Powell, G. V. N. Powell, H. O. R. Schubart \& M. B. Hays. 1986. Edge and other effects of isolation on Amazon forest fragments. Pp. 257-285. In: M.E. Soulé, (Ed.), Conservation Biology: the Science of Scarcity and Diversity. Sinauer Associates. Sunderland, MA.

Martín del Campo, R. 1953. Contribuciones al Conocimiento de la Herpetología de Nuevo León. Universidad, 11: 115-152.

Patterson, B. D., D. F. Stotz, S. Solari, J. W. Fitzpatrick \& V. Pacheco. 1998. Contrasting patterns of elevation zonation for birds and mammals in the Andes of southeastern Peru. Journal of Biogeography, 25: 583-607. 
Pomerantz, G. A., D. J. Decker, G. R. Goff \& K. G. Purdy. 1988. Assessing Impact of Recreation on Wildlife: A Classification Scheme. Wildlife Society Bulletin, 16: 58-62.

Pough, F. H., R. M. Andrews, J. E. Cadle, M. L. Crump, A. H. Savitzky \& K. D. Wells. 2004. Herpetology. Third Edition. Pearson, Prentice Hall. New Jersey.

Proyecto de Modificación a la Norma Oficial Mexicana NOM-059-SEMARNAT-2001. 2008. Protección ambiental-Especies nativas de México de flora y fauna silvestres-Categorías de riesgo y especificaciones para su inclusión, exclusión o cambio-Lista de especies en riesgo. Diario Oficial de la Federación, 5 de diciembre.

Rabb, G. B. 1956. A new Plethodontid Salamander from Nuevo Leon, Mexico. Chicago Natural History Museum, 39: 11-20.

Smith, H. M. \& E. H. Taylor. 1945. An annotated checklist and key to the snakes of Mexico. Bulletin United States National Museum, 187: 1-239.

Smith, H. M. \& E. H. Taylor. 1950. An annotated checklist and key to the reptiles of Mexico exclusive of the snakes. Bulletin United States National Museum 199: 1-253.

Smith, H. \& E. Taylor. 1966. Preface to the reprint of Herpetology of Mexico, 3 vols. Eric Lundberg. Ashton, Maryland.

Taylor, E. H. 1941. Two New Species of Mexican Plethodontid Salamanders. Proceedings of the Biological Society of Washington, 54: 84-86.

Treviño, C. H. 1978. Estudio herpetofaunístico distribucional del Sur de Nuevo León, México. Universidad Autónoma de Nuevo León, Facultad de Ciencias Biológicas. Monterrey, Nuevo León.

Werler, J. E. \& J. R. Dixon. 2000. Texas snakes: Identification, distribution and natural history. University of Texas Press. Austin, Texas.

Wilcove, D. S., C. H. McLellan \& A. P. Dobson. 1986. Habitat fragmentation in the temperate zone. Pp. 237-256. In: M.E. Soulé, (Ed.). Conservation Biology: the Science of Scarcity and Diversity. Sinauer Associates. Sunderland, MA. 